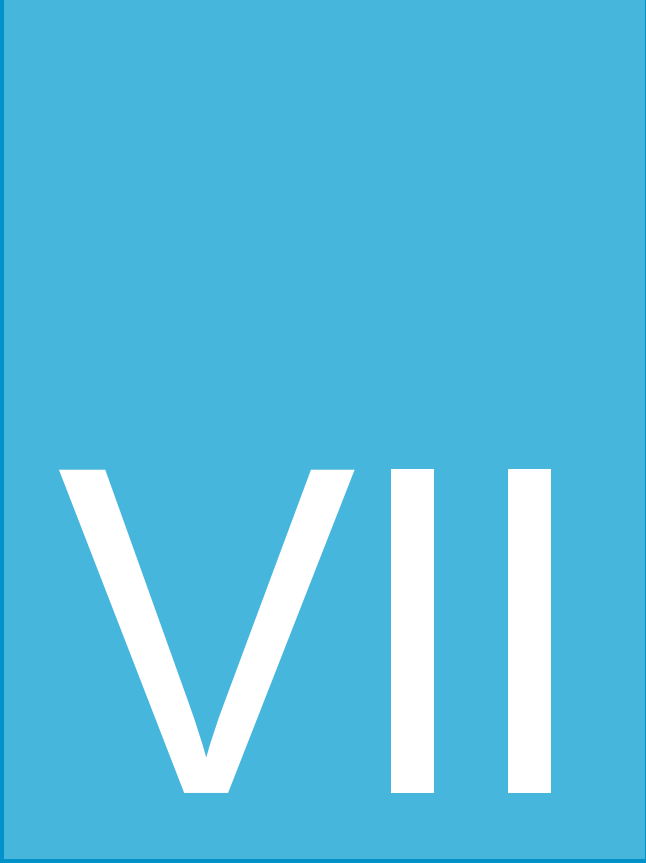

\title{
Breve historia de la educación en el Perú
}

\section{A brief history of education in Peru \\ Universidad Peruana Unión, Perú}

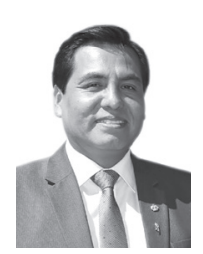

\section{Abel Apaza Romero}

Docente de la especialidad de Inglés-Lengua en la Universidad Nacional de Educación. Magister en educación con mención en Enseñanza del Idioma Inglés como Idioma Extranjero por la Universidad de Piura. Docente con experiencia en diversos niveles educativos. Desde el año 1999 hasta el año 2015 trabajó en la Universidad Peruana Unión como docente, director de la escuela de educación, también como secretario académico y decano encargado de la Facultad de Ciencias Humanas y Educación. En la actualidad se desempeña como Director de Educación y Libertad Religiosa en la Unión Peruana del Sur. 


\section{Resumen}

Esta revisión teórica busca identificar los factores clave de la educación peruana presentes en su calidad educativa frente a los países desarrollados, además se da a conocer la historia de la educación adventista en el Perú y sus aspectos fundamentales. Este estudio se realizó en base a obras realizadas por historiadores, artículos de analistas educativos y a textos de Ellen White. Se concluye que los factores clave para lograr el éxito económico y social de una nación se encuentra en la conexión entre la acción y el conocimiento, entre la investigación y a la formación de profesionales al servicio de las necesidades del Estado, una educación basada en el servicio al prójimo.

Palabras clave: Historia de la educación, acción y conocimiento, investigación, servicio, educación cristiana.

\section{Abstract}

This paper is a theoretical review and seeks to identify the key factors in the success achieved in the quality of education in developed countries, and also discloses the history of Adventist education in Peru and its fundamental aspects. This study was conducted based on works by historians, analysts educational articles and texts of the Spirit of Prophecy. It is concluded that the key factors for achieving economic and social success of a nation lies in the connection between action and knowledge, between research and the training of professionals serving the needs of the state, an education based on service neighbor.

Keywords: History of education, action and knowledge, research, service, christian education. 


\section{Introducción}

Al echar un vistazo a la historia de países del primer mundo, tales como Alemania, Suiza, Japón, Estados Unidos, y otros, podemos concluir que gran parte del éxito que ahora gozan depende de la idea de "la concepción de la educación vinculada esencialmente al trabajo productivo" de acuerdo con Pinilla (1966). Idea, la cual no dudaron en darle todo el impulso sobre todo después de quedar en la ruina después de la primera y segunda guerra mundial. La conexión entre la acción y el conocimiento, confirmaban la concepción de que el conocimiento debía servir como guía rector de la acción transformadora del mundo. Dicha conexión, muchas veces, en muchas partes de nuestro país, sobre todo en los pueblos marginados de nuestra sociedad, llega a ser un mito. Un mito que es cada día abatido con cada frustración de los padres al ver el resultado en sus hijos sin el "cambio transformador" que esperaban. White (1963) aseveraba:

Los alumnos deben salir de nuestros colegios dotados de eficiencia cabal, para que cuando dependan de sus propios recursos, tengan conocimientos que puedan usar, útiles para tener éxito en la vida. Es esencial el estudio diligente, pero también lo es el trabajo arduo y laborioso. El juego no es esencial. El dedicar las facultades físicas a la diversión no es lo más favorable para tener una mente bien equilibrada. Si el tiempo empleado en el ejercicio físico que paso a paso conduce al exceso, fuese dedicado a trabajar de acuerdo con los métodos de Cristo, la bendición de Dios descansaría sobre el obrero. La disciplina para la vida práctica que se obtiene del trabajo físico combinado con el esfuerzo mental, queda endulzada al reflexionar que ella hace a la mente y al cuerpo más idóneos para cumplir la obra que Dios requiere que los hombres hagan. Cuanto más perfectamente sepan los jóvenes cumplir los deberes de la vida práctica, tanto mayor será el gozo que tendrán día tras día por ser útiles a otros. La mente educada para disfrutar del trabajo provechoso se amplía; por la preparación y la disciplina se hace idónea para ser útil; porque adquiere el conocimiento esencial que permite a su poseedor beneficiar a otros.

Veamos algunos antecedentes históricos de la educación en el Perú:

\section{El Incario}

El Perú tiene tradición de nobles esfuerzos educativos y laborales que hicieron posible el desarrollo en la época de la cultura preincaica e incaica y durante la colonia y el virreynato. 
En el Imperio incaico no existían escuelas generalizadas. Los bienes culturales fundamentales eran transmitidos en la familia y en la comunidad de modo natural. Un tipo de educación superior debió impartirse en el Cuzco, en el llamado "Yachayhuasi" o casa del saber. Su acceso estaba restringido a la joven nobleza imperial...del sexo femenino, sólo las escogidas o "aclias" recibían instrucción sistemática (Kauffmann, 1963). El Amauta era el "verdadero científico", profesional del conocimiento, director de la investigación y de la acción. El no sólo era dueño del acervo de verdades que se conservaba en la comunidad y se transmitía, como una herencia cultural, de unas generaciones a otras...era también inventor, creador (Valcarcel 1943).

El desarrollo del imperio incaico no solo se debió a la importancia de la educación sino también a la ética, ("no robarás, no mentirás, no seas perezoso"). Podemos ver, como el intelecto, el trabajo y la ética fueron elementos indispensables para el notable desarrollo de uno de los mayores imperios en el mundo antiguo. Sin embargo, existía una diferenciación de clases beneficiando a las clases nobles.

\section{La Colonia}

El Estado Iglesia español, fue el gran actor de la conquista y la colonización, justificándose en los valores espirituales, y que estos valores exigían la integración humana entre españoles y nativos. Las órdenes religiosas de los dominicos, de los franciscanos, y de los jesuitas, que realizaron la tarea educativa a los niveles primario, secundario y técnico, influyendo sino en toda la sociedad.

Gracias al esfuerzo de los jesuitas, se expande la civilización gradualmente en la zona de la cuenca amazónica. Fueron los jesuitas los que evangelizaron el Japón en el siglo XVII y los primeros occidentales que establecieron una comunicación efectiva y pacífica con el Celeste Imperio de la China (Fulop 1931).

La grandeza de esta obra colonizadora se conservó aún en los primeros años de la república y el proceso de desarrollo alcanzado en ese tiempo resalta frente al subdesarrollo en el que la misma época se encontraban países como Japón y Estados Unidos.

El historiador norteamericano Moehlman (1957) afirma que es una errada concepción ampliamente extendida el pensar con respeto a la educación en Latinoamérica que los conquistadores tuvieron poco interés en la educación y que no hubo escuelas públicas con anterioridad al período de la independencia. Nada podía estar más alejado de la verdad. Uno de los más notables aspectos del esfuerzo colonial en muchas partes de Latinoamérica fue la tremenda cantidad 
de energía y fondos gastados en el establecimiento de escuelas - desde escuelas para infantes hasta universidades. En muchas instancias estas escuelas estaban especialmente interesadas en las poblaciones de mestizos nativos. En algunos casos las escuelas eran exclusivamente para europeos. En algunas áreas las escuelas eran primariamente para adiestrar miembros del clero, en otras, el propósito educacional era más amplio.

Nadie duda, que los conquistadores no tuvieron interés en la educación, la mayor prueba de ello la tenemos en la Universidad Nacional Mayor de San Marcos, la primera universidad en América. Sin embargo, podemos preguntar: ¿Quiénes se beneficiaban con dicha educación? ¿Podían, acaso, los “indios" tener acceso a dicha educación?

Valcárcel (1972), describe de una manera magistral una experiencia que puede resumir el espíritu de servicio abnegado que siempre imperó en los misioneros adventistas.

El adventista estaba ayudando al alumbramiento del nuevo indio. Su asepsia se deja ver en la extirpación de los vicios seculares: alcoholismo, cocainismo, servilismo. El hombre que en la altipampa del Collao representa hoy la tradición milenaria del Tiahuanaco, posee hábitos higiénicos, viste de americana, reside en limpias moradas, no bebe aguardiente ni pijcha coca: es abstemio. $\mathrm{Ha}$ aprendido a mirar de frente, a hablar con aplomo y a extender la mano en gesto amistoso a cuantos favorece con su simpatía. Es un hombre.

La obra adventista adquiere enormes proyecciones. La Escuela Normal de Juliaca, el crecido número de escuelas y maestros que sostiene, la organización en gran escala de sus oficinas, todo revela que estamos en presencia de un poder social nuevo que no por negarlo deja de existir y que -por lo contrario- debe ser atentamente examinado para conocer sus métodos y denunciar sus peligros.

Valcarcel resalta la importancia del trabajo de la Iglesia Adventista del Séptimo Día en la región de Puno, llevando la educación a una población abandonada por las clases sociales altas.

Si bien en el imperio incaico ya existía una diferenciación de clases, al beneficiar a la clase noble, en la época de la colonia fue mucho más radical al cerrar totalmente el acceso a la lectura y escritura a nuestros antepasados. En este contexto, es notable resaltar el trabajo que realizó el pionero Fernando Stahl al iniciar una gran labor educativa en 1911 con la gente marginada del altiplano peruano. Maquera (2014), afirma que, 
...para Stahl la realidad del habitante de los Andes peruanos no era ajeno, en sus visitas anteriores tomó conocimiento de la situación educativa, social, económica y de salud de sus moradores, él sabía a dónde estaba llegando, conocía de los abusos más desalmados que los campesinos sufrían. Stahl describe cómo encontró a los campesinos y expresa en una sola frase su percepción: "una raza oprimida.

Esto trajo consigo una lucha, por parte de las clases marginadas y explotadas, por las poblaciones campesinas, por los más pobres, por querer alcanzar esa privilegiada educación. El poder leer y escribir, era una victoria, era alcanzar un status social en donde ya no llegarían a ser más explotados. Dicha lucha, felizmente, a pesar de las diversas decepciones de la escuela, la podemos ver aún en aquellas clases sociales, las cuales todavía están bajo una explotación no tan sutil: la de la sociedad.

A través de la historia podemos ver, como es que las clases sociales altas podían gozar de ese privilegio llamado educación. Sin embargo, podemos preguntarnos:

¿Qué sucedió entonces? ¿Qué retrasó el ritmo de desarrollo en Latinoamérica y sin embargo aceleró, el desarrollo educativo y cultural, económico y político de los Estados Unidos y el Japón?

Estados Unidos optó por las universidades dedicadas a la investigación científica y a la formación de profesionales al servicio de las necesidades del Estado.

Japón, a través de la influencia de los Estados Unidos, estableció también con éxito estos sistemas educativos que desataron su desarrollo.

En Latinoamérica, debido a la influencia ideológica de Francia, especialmente, y de las mal comprendidas ideas del enciclopedismo y de sus miopes aplicaciones pedagógicas, se paralizó la acción educadora del Estado Iglesia. El respaldo que la corona prestaba a la educación pública no fue eficazmente sustituido por los nuevos Estados democráticos emergentes (Pinilla 1966).

Definitivamente la realidad peruana es mucho más compleja de describir. Sin embargo, este hecho explica en parte el porqué, mucha gente se decepciona del mito que indica que la persona educada es más útil a la sociedad. Ya que la escuela preparaba (y continua preparando) alumnos no muy útiles a la sociedad, no productivos, debido a los modelos educativos implantados, los cuales estaban lejos de nuestra realidad. 


\section{La República}

Pinilla (1966) nos narra la historia de la educación peruana durante el periodo de la republica al citar a San Martín quién puso énfasis en la instrucción pública como la primera necesidad de las sociedades y consideró la integración de las poblaciones indígenas, a quienes, dijo no debe llamarse "indígenas" sino "peruanos". Ramón Castilla realizó una obra de estructuración, organización e integración nacional, basada en el profundo respeto a la ley, a las instituciones jurídicas y a los ideales nacionales. Concibió a la educación como el instrumento esencial para el desarrollo nacional, dictando la Ley Orgánica de Enseñanza.

Santa Cruz creó la Dirección General de estudios, mientras que el gobierno de Prado creó una Inspección General de Instrucción Primaria.

No faltaron hombres que tuvieron ideas claras respecto a la naturaleza del esfuerzo educativo, y que sin embargo sus voces no fueron escuchadas. Este mismo hecho aún lo podemos ver actualmente, en nuestra realidad.

\section{Siglo XX}

Trahtemberg (2011) hace una descripción breve de la historia de la educación peruana durante el siglo $\mathrm{XX}$, el cual se inicia con esfuerzos gubernamentales por incrementar sustantivamente el número de estudiantes con acceso a las escuelas primarias, en circunstancias en las que la educación era concebida como el medio capaz de producir la integración nacional, el "amor y lealtad a la patria" y el desarrollo económico, con los que se lograría el ascenso social de los indígenas y la igualdad entre peruanos sin distingo de fortunas.

Fue el gobierno de José Pardo (1904-8) el que se delineó y puso en práctica una primera reforma educativa de envergadura que ordenó y organizó lo pre existente y le dio un norte para los desarrollos futuros. Impulsó la educación primaria procurando universalizarla, estableciendo la gratuidad y obligatoriedad de la misma, incluyendo el reparto gratuito de material de enseñanza. También estableció la gratuidad de las escuelas técnicas y ocupacionales nocturnas, recuperando las experiencias de las escuelas de Artes y Oficios pre existentes hasta 1902. Además le dio un particular impulso a la educación privada. Todo esto se hizo con el apoyo de pedagogos franceses, belgas y especialmente alemanes.

Es en esta década en dónde inicia la educación adventista en el Perú, Castillo (2010), señala que 
...hasta donde ha sido posible auscultar, y por curioso, raro o ingenuo que parezca, las raíces de la Universidad Unión Incaica se remontan hasta el día viernes 15 de marzo de 1918, día y fecha cuando el Concejo Provincial de Lima visó el Libro de Matrícula de la Escuela Libre que funcionaba en la Calle San Francisco (Plazuela) No. 350 (hoy Jirón Ancash), fundada y dirigida por el joven normalista don Fernando F. Osorio, inicio, del que más tarde sería el Colegio Unión localizado en Miraflores y posteriormente, en 1946, trasladado a Ñaña.

Con Leguía se estableció una primaria común gratuita y obligatoria y otra profesional; una secundaria común obligatoria y otra profesional; y la superior que incluyó las Escuelas Técnicas de Ingeniería y Agricultura, la Universidad Mayor de San Marcos y las Universidades Menores de Cuzco, Arequipa, Trujillo, así como la Universidad Católica creada en 1917. En 1921 los pedagogos de la misión pedagógica de EE.UU. asumieron la Dirección General y las Direcciones Regionales del Ministerio de Educación lo que significó el giro del enfoque intelectualista, academicista e integral europeo al enfoque norteamericano más pragmático y articulado con el desarrollo económico.

En los años 1940's el gobierno de Prado le dio un gran impulso la educación técnica y normal y además estableció la educación infantil para niños de 4 a 7 años, la primaria común gratuita y obligatoria de 6 años, la secundaria común de cinco años con ingreso a la opción técnica, normal o artística al cabo del 3ero de secundaria y la superior. Se abrieron las puertas del magisterio a las clases medias que le imprimieron a la carrera magisterial un nuevo rostro social. Ya no era una profesión para las élites sino un empleo remunerado de profesionales oriundos de los sectores medios al servicio de las amplias capas populares.

En 1945 Bustamante y Rivero estableció la gratuidad de la secundaria para los alumnos egresados de las escuelas fiscales, lo que trajo el inicio de la expansión de la secundaria aunque sin contar con los profesores e instalaciones adecuadas. Esta fue una época de transición demográfica del campo a la ciudad, de grandes migraciones alimentadas por la escuela urbana y mestiza, que se convertiría en factor de integración al alfabetizar y castellanizar a los migrantes, pero a la vez se constituiría en factor de exclusión por motivos étnico culturales. Para atender esta creciente demanda de educación secundaria gratuita el gobierno de Odría aprobó su "Plan Nacional de la Educación" por el que se crearon 55 Grandes Unidades Escolares. Nuevamente se imitó los modelos norteamericanos que procuraban mejorar la enseñanza de las ciencias y expandir la educación técnica. Además creó colegios secundarios militares con alumnos internados egresados del 3er año de secundaria común. También creó 8 nuevas 
universidades, reabrió Huamanga y permitió la creación de otras 7 universidades privadas.

En 1958 con Prado se abrieron las opciones de "ciencias" y "letras" para la secundaria y en 1962 creó el SENATI para responder a las crecientes necesidades de formación de técnicos de mando medio y mano de obra calificada en un ambiente de sustitución de importaciones y desarrollo de la manufactura nacional. Esa misma fecha, los sindicatos de docentes de Primaria, Secundaria, Técnica y Educación Física se articularon en la "Federación Nacional de Educadores del Perú" (FENEP) que luego en 1972 daría origen al "Sindicato Único de Trabajadores en la Educación del Perú" (SUTEP).

El gobierno de Belaunde impulsó la planificación educativa, elevó el atractivo de la carrera docente con un nuevo escalafón a la vez que elevó las remuneraciones. Estableció la gratuidad de toda la enseñanza estatal ampliando la cobertura de primaria y secundaria presionando además el ingreso a la superior, masificándose las "academias" de ingreso a las universidades y las escuelas normales. Sin embargo, se redujo la jornada escolar y se deterioró la calidad de los docentes.

La Reforma Educativa de Velasco en 1972 abordó la educación como una cuestión política y económica-social, que debía comprometer la participación organizada de la comunidad vinculándola con el desarrollo y el trabajo y planteaba que no se podía cambiar la estructura económica, social y cultural del país si no se reformaba su educación, y no se podía transformar su educación si no se modificaban las bases económicas y sociales sobre las cuales estaba sustentada. A falta de recursos, la mayor demanda educativa no se cubrió con nueva infraestructura sino con un recorte de la jornada escolar, aumento de turnos escolares en los mismos locales y aumento de número de estudiantes por profesor.

Recuperada la democracia en 1980, el 2do gobierno de Belaunde desactivó las propuestas reformistas velasquistas, regresando al esquema de educación primaria y secundaria, transformando los núcleos en Supervisiones Educativas (que luego se transformarían en Unidades de Servicios Educativos USE). También promulgó una nueva Ley del Profesorado (24029).

Durante el Gobierno de Alan García (1985/90) se hizo un esfuerzo de consulta nacional y elaboración de un "Proyecto Educativo Nacional" que reflejara las necesidades de la educación peruana.

Durante esta década se marca un hito histórico para la educación adventista en el Perú al crearse la primera Universidad Adventista en Sudamérica, la Universidad Unión Incaica, hoy Universidad Peruana Unión. Castillo (2010) narra este momento:

Revista Apunt. Univ. VOLUMEN VI • NÚMERO 2 p. 111 - 124 
La creación de la Universidad Unión Incaica fue consignada como la Ley No. 23758, aprobada el 15 de diciembre en la Cámara de Senadores; firmada en el Congreso el 22 de diciembre por Ricardo Monteagudo, Presidente de la Cámara de Senadores y Dagoberto Lainez Vodanovic, Presidente de la Cámara de Diputados; promulgada el 30 de diciembre por el Arq. Fernando Belaúnde Terry, Presidente Constitucional de la República, siendo Patricio Ricketts Rey de Castro Ministro de Educación; y publicada por el diario oficial El Peruano el 31 de diciembre de 1983, para que entre en vigencia el 10 de enero de 1984...

En 1990 asumió el gobierno Alberto Fujimori, quién jaqueado por los retos del terrorismo que además estaba infiltrado en un sector del magisterio, osciló entre propuestas municipalizadoras y privatizadoras de la educación, y la defensa de la educación pública gratuita. En su primer quinquenio de gobierno, el gobierno se dedicó fundamentalmente a mejorar e incrementar la infraestructura y a revalorizar la educación pública gratuita incluyendo en ella los alimentos, el seguro escolar, la instalación de computadoras, así como la capacitación masiva de profesores. Sin embargo, vencido el terrorismo continuó el régimen centralista, burocratizado y reglamentarista, el cual solo alentó la expansión de la educación privada en todos los niveles. Así la oferta educativa privada creció significativamente, sobre todo en el nivel superior. Sin embargo, no se ensayaron fórmulas descentralizadoras.

Trahtemberg (2011), afirma que durante este siglo encontramos como constante el centralismo controlista, y sumado a ello tenemos el impulso a la educación estatal gratuita. El financiamiento per cápita, cada vez menor, aunque con un período de bonanza entre los años 1950's y 1960's, asociado con el descenso de la calidad de la educación, estuvo unido al deterioro de las condiciones de vida y profesionales de los docentes. Esto ocurrió porque en lugar de acompañar la expansión de la cobertura educativa con la paralela expansión del presupuesto destinado a la educación para evitar afectar la inversión educativa per cápita, se redujo el presupuesto y las jornadas escolares, se aumentó los turnos por colegio y el número de estudiantes por profesor y se redujeron las remuneraciones docentes. Obviamente esto disminuyó el atractivo de la carrera docente que se debilitó como profesión, y a su vez llevó a los docentes a organizarse en un poderoso sindicato único que con el tiempo fue radicalizando sus demandas y protestas. Al deteriorarse la calidad de la educación pública, se incrementó la demanda por la educación privada.

El estado ha luchado por visualizar, diseñar e implementar un modelo educativo peruano que responda a los requerimientos y posibilidades peruanas. 
Hemos sido permanentes copiadores de modelos extranjeros y hemos pasado de estar en manos de las misiones belgas, alemanas y norteamericanas, a depender de las prioridades establecidas por los organismos de cooperación técnica y financiera internacionales.

Durante el siglo XX encontramos altos y bajos en el financiamiento de la educación, en las propuestas reformistas y en la calidad de los profesores unido a la falta de consistencia y continuidad de las propuestas las que fueron debilitadas y diluidas con un consecuente deterioro, con un notorio retraso frente a los países líderes y con brechas educativas equivalentes a las que tuvimos a principios de este siglo.

\section{Siglo XXI}

Durante los gobiernos de Valentín Paniagua y Alejandro Toledo (2000 al 2006) la economía se estableció creciendo a un ritmo inusitado. Se creó una nueva Ley General de Educación, sin embargo

De acuerdo al Ministerio de Educación (2016), en la actualidad, en el Perú hay 470, 635 profesores de educación básica regular pública que atienden a más de dos millones de alumnos; sin embrago, el $75 \%$ de estos se encuentra en la ciudad y solo el $25 \%$ en zonas rurales, que es donde más hacen falta. A esta problemática, también se suma el bajo sueldo que perciben los educadores en nuestro país, que a diferencia de los profesores de otros lugares de la región, perciben mucho menos por las horas pedagógicas. Una de las principales necesidades para mejorar el nivel educativo es la capacitación permanente de los profesores y por ello el Ministerio de Educación invierte 689 millones de soles al año en soporte pedagógico, talleres, cursos y programas de actualización. Para el presente año se tiene previsto capacitar a 185 mil docentes con un presupuesto destinado de 573 millones de soles.

\section{Conclusión}

El mito de la educación se puede subdividir en otros mitos dependiendo desde donde es visto. Así tenemos el mito del estudiante, él era (y tercamente lo es, en la actualidad) aquella persona la cual tenía la obligación de satisfacer los requerimientos del profesor. No interesaba su opinión, ni sus gustos, considerándolo incapaz de poder tener esa competencia de decisión.

El mito del maestro, lamentablemente todavía se puede ver en la 
sociedad peruana, a maestros, que piensan que los estudiantes están para servirle, teniendo un lugar muy alejado del estudiante y de su realidad.

El mito de la escuela, lugar en donde aquel quien ingresa, sale con una capacidad superior, capaz de transformar la sociedad y de poder alcanzar un status superior.

Muchos de estos mitos, aún están muy arraigados a nuestra sociedad, sin embargo una buena parte de la población piensa diferente.

Villaran (1962) nos dice:

El Perú debería ser por mil causas económicas y sociales, tierra de labradores, de colonos, de mineros de comerciantes, de hombres de trabajo; pero las fatalidades de la historia y la voluntad de los hombres han resuelto otra cosa, convirtiendo al país en centro literario, patria de intelectuales y semillero de burócratas. Somos un pueblo donde ha entrado la manía de las naciones viejas y decadentes, la enfermedad de hablar y escribir y no de obrar, de agitar palabras y no cosas, dolencia lamentable que constituye un signo de laxitud y de flaqueza.

Luis Alberto Sánchez (1963) dice con razón que la gran cuestión pedagógica del Perú consiste en la educación del niño serrano. Ya que las grandes mayorías nacionales están conformadas por niños menores de 14 años y que la población indígena y serrana del Perú es la mayor. Tiene razón también al poner énfasis en que la educación no consiste exclusivamente en enseñar a leer sino en interesar al niño por resolver inteligentemente los retos y los problemas que le plantea su medio originario para lo que es menester que siga pensando, siga leyendo y siga estudiando. Así el niño aprenda no solo a leer sino que siga leyendo y se oriente en la vida por medio de un oficio y de cierto sistema general de ideas sobre el mundo y el país donde nació y subsiste.

Quienes nos dedicamos a la educación, tenemos muy claro que pueden lograrse muchas mejoras en los niveles de aprendizaje y motivación en los estudiantes. Todos tenemos anécdotas formidables de actividades significativas y motivantes. Hemos conocido colegas cuyas clases frente a una clase nos dejan sorprendidos por la sencillez y eficacia con que logran la comunicación y la didáctica. Si todo esto es cierto, porque lo vemos cotidianamente, ¿ipor qué razón entonces los indicadores internacionales y nacionales de eficacia educativa muestran un deterioro sostenido en América Latina en lo general y en numerosos países del mundo? (UNESCO, 1997). 
A pesar de los grandes esfuerzos de nuestros gobiernos por definir políticas educativas vanguardistas, el profesor sigue esperando que suceda algo que pueda alterar los escenarios que muestran una realidad educativa con resultados alarmantes.

De acuerdo con Coombs (1986), las suposiciones básicas (mitos) que dieron lugar a la crisis actual educativa son las siguientes:

- La suposición de que "La educación formal universal para todos los niños y jóvenes y el logro de la alfabetización para todos los adultos erradicaría las desigualdades sociales y económicas profundamente arraigadas de todas las sociedades".

- La suposición de que la "expansión de la educación sería el carburante del crecimiento económico mundial".

- Y la más grave de todas las suposiciones es creer que "los sistemas educativos existentes, heredados del pasado, estaban bien adaptados para responder a las necesidades y metas nacionales de la situación tan transformada de la posguerra".

Desde un punto de vista cualitativo Coombs señala que "la crisis educativa mundial es, en esencia, una crisis de creciente desadaptación entre los sistemas educativos heredados y el mundo rápidamente cambiante de nuestro alrededor". El Perú no está ajeno a esta realidad. Trahtemberg (2000), afirma que,

El Perú tiene que reinventar creativamente sus propuestas educativas porque no tenemos el tiempo y el dinero para recorrer el mismo camino que otros países recorrieron para desarrollar su educación. Debemos inventar las recetas educacionales novedosas, concebir cruces de caminos originales que permitan saltar etapas, colocar los escasos recursos en proyectos con enorme efecto multiplicador y utilizar tecnologías que tengan gran cobertura y que potencien las limitadas capacidades docentes que exhiben la mayoría de los profesores peruanos. Este es el reto para el inicio del siglo XXI.

Como maestros, sabemos muy bien que no podremos cambiar, desde nuestro puesto, la forma de dirección de nuestros gobernantes, tampoco podremos cambiar la forma de pensar de nuestros administradores, tampoco podremos cambiar la manera de pensar de nuestros colegas. Sin embargo, podemos cambiar nuestra actitud, nuestra influencia, teniendo el reto de 
reconvertirnos, de ser flexibles para admitir el cambio y aceptar las corrientes de pensamiento y de tecnología que seguramente repercutirán en la forma en que hacemos educación. Esa conexión entre la acción y el conocimiento es posible poder desarrollarlo desde nuestro accionar y así poder contribuir con el desarrollo de nuestra sociedad.

\author{
Abel Apaza Romero \\ Universidad Peruana Unión, Perú \\ email: abelapazar@gmail.com \\ Recibido: 12 de febrero de 2016 \\ Aceptado: 22 de abril de 2016
}

\title{
Referencias
}

Ansión J. (1995). El Perú frente al siglo XXI. P.U.C.P.

Ansión J., Lazarte A., Matos S., Rodríguez J. y Vega J. (1998) Educación: La Mejor Herencia. P.U.C.P.

Cadwallader E. (2010). Principios de la educación adventista en el Pensamiento de Elena de White. Filosofía, Objetivos, Métodos y Misión. Lima: Fondo editorial Universidad Peruana Unión.

Castillo R. (2010) La mano de Dios en la educación adventista del Perú. Breve historia de la creación de la UPeU. Lima: Fondo editorial Universidad Peruana Unión.

Fulop M. (1931). El Poder y los secretos de los Jesuitas. Biblioteca nueva, Madrid.

Kauffmann F. (1963). Los Incas y el Tahuantinsuyo. Peruanistica.

Maquera J. (2014). Operación Andes libres construyendo la "Utopía”... Lima: Fondo editorial Universidad Peruana Unión

Moehlman y Roucek (1957). Comparative Education. The Dryden Press, New Cork.

Phillip H. (1986). "Crisis en la educación superior", Fundación Para la Educación Superior, Bogotá, Colombia.

Pinilla A. (1966). Educación para el desarrollo nacional. Universidad de Lima.

Radio Programas del Perú (2016). Los docentes en la educación pública. Recuperado de http://rpp. pe/politica/elecciones/asi-esta-el-peru-2016-los-docentes-en-la-educacion-publicanoticia-939152

Reyes A. (1997). Técnicas y modelos de calidad en el salón de clases. Trillas.

Sánchez L. (1963). El Perú: Retrato de un país adolescente, U.N.M.S.M. Lima Perú.

Trahtemberg (2000). Evolución de la educación peruana en el siglo XX. http://www.trahtemberg.com/ articulos/1169-evolucion-de-la-educacion-peruana-en-el-siglo-

UNESCO (1997). "Descuido a maestros, ONU”. Diario El Norte.

Valcarcel L. (1943). Historia de la cultura antigua del Perú. Tomo I vol. II. Lima.

Villaran, M. (1962). Páginas escondidas. P.L. Villanueva. 1962

White E. (1963) La educación cristiana. Casa Editora Sudamericana. 